\title{
A Novel and Effective Acupuncture Modality as A Complementary Therapy to Acute Pain Relief in Inpatients with Rib Fractures
}

\author{
Hsin-Yi Ho ${ }^{1}$, Chao-Wei Chen ${ }^{1}$, Ming-Chieh Li ${ }^{2}$, Yu-Pao Hsu ${ }^{3}$, Shih-Ching Kang ${ }^{3}$, Erh-Hao Liu ${ }^{3}$, \\ Ko-Hung Lee ${ }^{4}$
}

Background: Pain control has been emphasized as a priority for both practitioners and inpatients with rib fractures, since analgesia could only offer limited relief from severe pain. A prospective and randomized controlled trial was conducted to analyze the efficacy and efficiency of acupuncture in acute pain relief for inpatients with rib fractures.

Methods: A total of 58 inpatients were recruited and allocated to two groups, receiving identical doses of conventional oral analgesics as well as filiform needles as treatment and thumbtack intradermal (TI) needles placed upon the skin surface as a control, respectively, via novel acupuncture modality once daily for three consecutive days. The effect of pain relief was evaluated during activities that induce pain, and sustained maximal inspiration (SMI) lung volumes and sleep quality were assessed.

Results: The patients treated with filiform needles had more effective pain relief than those in the TI needle group during deep breathing, coughing, and turning over the

\section{At a Glance Commentary}

Scientific background of the subject

Rib fractures are the most common type of injury in chest trauma. Inpatient rib fracture pain control has been emphasized from both pulmonary and analgesia standpoint, and there still exists room for better therapy.

\section{What this study adds to the field}

This innovative acupuncture modality could be considered as a complementary therapy to oral analgesia to relieve rib fracture pain. Pain intensity assessed during pain-inducing actions may impact further studies concerning pain relief via acupuncture. body $(p<0.05)$, and the effect persisted for at least $6 \mathrm{~h}$ in most patients. Sustained maximal inspiration lung volumes and sleep quality did not show improvement through every acupuncture intervention, and they could not respond accurately to pain relief via acupuncture.

Conclusion: The active evaluation could provide a more adaptive model for assessing pain intensity due to rib fractures. This novel acupuncture modality in which the needle insertion sites are corresponding to the pain spots can be a safe and viable therapy for relieving pain in inpatients with rib fractures.

(Biomed J 2014;37:147-155)

Key words: acupuncture, acute pain, pain relief, rib fractures

$\mathrm{R}$ ib fractures, which are usually caused by traffic accidents and falls, are among the most common injuries in blunt thoracic trauma. ${ }^{[1-3]}$ The main symptom is intense flank pain, which may last for at least 1 month and impede the functions of deep breathing, coughing, or turning over in bed of the patients ${ }^{[1,4]}$ and, therefore, is associated with the occurrence of pneumothorax, hemothorax, atelectasis, and pneumonia. ${ }^{[3,5]}$ Also, pain in patients with rib fractures

From the ${ }^{1}$ Department of Chinese Orthopaedics and Traumatology, Chang Gung Memorial Hospital at Taoyuan, Chang Gung University College of Medicine, Taoyuan, Taiwan; ${ }^{2}$ Institute of Occupational Medicine and Industrial Hygiene, College of Public Health, National Taiwan University, Taipei, Taiwan; ${ }^{3}$ Department of Trauma and Emergency Surgery, Chang Gung Memorial Hospita at Linkou, Chang Gung University, Taoyuan, Taiwan; ${ }^{4}$ Department of Electrical Engineering, Chang Gung University, Taoyuan, Taiwan

Received: Apr. 05, 2013; Accepted: Jun. 18, 2013

Correspondence to: Dr. Ko-Hung Lee, Department of Chinese Orthopaedics and Traumatology, Chang Gung Memorial Hospital at Taoyuan. 123, Ding-Hu Rd., Jiou-Lu Tsuen, Gueishan, Taoyuan 333, Taiwan (R.O.C). Tel: 886-3-3196200 ext. 2613; Fax: 886-3-3298995; E-mail: greglkh@yahoo.com.tw

DOI: $10.4103 / 2319-4170.117895$ 
usually leads to increased mortality in older population and prolonged disability. ${ }^{[1,2,6-9]}$

Acute pain management in inpatients with rib fractures has been highly emphasized by practitioners. The commonly used oral analgesics, including nonsteroidal anti-inflammatory drugs (NSAIDs), seem to provide limited relief from severe pain. ${ }^{[1,10]}$ Rib taping and other invasive modalities such as intercostal nerve block and operative repair have also been practiced to relieve the pain due to rib fractures, but still some controversies have been shown over the benefit and their indications. ${ }^{[1,9-12]} \mathrm{A}$ novel and effective therapy for relieving pain from fractured ribs is, therefore, inevitably needed for both physicians and patients.

Pain control via acupuncture has been widely investigated and acknowledged, and broadly applied in several conditions such as low back pain, osteoarthritis of knee, cancer, preoperative and postoperative pain.$^{[13-16]}$ However, there have been no clinical trials conducted to address the issue of easing rib fracture pain with acupuncture to date.

The objective of this pilot study was to analyze the efficacy and efficiency of acupuncture in acute pain relief for inpatients with rib fractures, focusing on activities inducing pain such as deep breathing, coughing, and turning over in bed. Sustained maximal inspiration (SMI) lung volumes and quality of sleep were also evaluated. A novel and individualized acupuncture therapy based on our clinical experience was performed in this study with two kinds of acupuncture instruments, filiform needles and thumbtack intradermal (TI) needles. We anticipate this new model of acupuncture plus pharmacological treatment to be developed to benefit patients with rib fractures in their treatment.

\section{METHODS}

\section{Study design}

This single-blind, prospective, randomized controlled, phase II clinical trial was conducted at the Chang Gung Memorial Hospital at Linkou in Taiwan. The study was approved by Institutional Review Board of Chang Gung Medical Foundation (IRB number: 100-3116A3) and was performed from 8 December 2011 through 8 November 2012. A table of random numbers was adopted to ensure the randomized allocation to two groups, filiform needle (FN) group and control group, in a 1:1 ratio. The generator of randomized table, the evaluator who collected the outcome measure, and the statistician who performed the final analysis were all blinded to the grouping.

\section{Participants}

The participants were recruited from the inpatients diagnosed with rib fractures with chest X-ray in the Department of Trauma and Emergency Surgery at the hospital. One hundred and thirty-five inpatients with rib fractures were screened, with 92 of them meeting the inclusion criteria, and 58 of them participated in the study with written informed consent and underwent randomization. The baseline characteristics such as lesion sides, etiologies of rib fractures, number of fractured ribs, body mass index (BMI), Injury Severity Score (ISS), and initial pain intensity after trauma of these participants were recorded. The same oral analgesia, ibuprofen (400 mg/6 h, four times a day), was prescribed for all the patients since admission.

Inclusion criteria included: (1) age 18 years and older; (2) presence of one or more, unilateral or bilateral rib fractures diagnosed with chest X-ray; (3) maximal rib pain score of more than 5 obtained using the numerical rating scale (NRS: 0-10) after patients took ibuprofen and then did any one of the following three actions: deep breathing, coughing, or turning over; and (4) ability to describe the sites of pain and evaluate the intensity accurately. Those patients who: (1) could not be examined for the movements of deep breathing, coughing, and turning over in bed; (2) had open wound on abdomen and lumbar region; (3) had any poorly controlled diseases such as atelectasis, pneumonia, or other infectious diseases, immune system dysfunction, bleeding tendency, psychiatric disorders, and skin problems; or (4) were pregnant were excluded from the study.

All the participants were told that they would receive acupuncture treatment in addition to the original oral analgesics and assess the efficacy of acupuncture. The patients were randomly assigned to two groups and blinded after providing written informed consent, without being mentioned which kind of needles were applied as control. The participants received no financial benefit from the study and were fully aware of their rights to withdraw at any time.

\section{Interventions}

Regardless of treatment groups, the same procedure of acupuncture regimen was performed by a certified physician who is also the only person in this trial aware of the group assignment. This physician did not take charge in randomization, outcome measurement, or statistical analysis, and had no interactions with study subjects after practicing acupuncture.

A specific acupuncture modality was performed at the patients' bedside, which orientated the acupuncture points with reference to different pain spots [Figure 1]. First, the patient was asked to find and point at the severest pain spot during deep breathing, coughing, or turning over. This pain spot was taken as an origin of the $Y$-axis which was parallel with the central line of trunk through the navel. The transverse line around the abdomen and lumbar region through the navel was regarded as the $X$-axis. The intersection of these two axes was the acupuncture point, located in abdomen or lumbar area, into which the needle was inserted 
after sterilization with alcohol cotton. After one needle was positioned, the same procedure above was practiced to find another acupuncture point. In each regimen, every patient received five needles which were fixed with opaque adhesive tape and retained for $6 \mathrm{~h} / \mathrm{day}$. All patients were told that these needles could be removed earlier if they felt any discomfort.

The patients were treated once daily for three consecutive days since the day of enrolment. The participants who could not complete the full treatment of three regimens were dropped out from the statistical analysis.

The only difference between the FN group and the control group was the intervention device. The FN group was treated with disposable, sterile, 2.5 -cm-long and 30-gauge filiform needles. The filiform needles were inserted at an angle of 15 into the surface of the skin at each point, and the total inserted length was $2 \mathrm{~cm}$. The control group was treated with disposable, sterile, 0.2 -cm-long TI needles. The TI needles were only placed on the surface of the skin, and their stimulation length was shallower than the filiform needles. The participants in both groups could feel the sting sensation from the two kinds of needles. The De Qi sensation was not emphasized in this acupuncture modality.

During hospitalization after joining this trial, the subjects could still receive other complementary management such as laxative agent and the original underlying medicine according to their condition.

\section{Outcome measures}

The primary outcome was effective pain relief, with maximal pain intensity (NRS: 0-10) evaluated by participants themselves during deep breathing, coughing, and turning over in bed in sequence before and 10 min after every

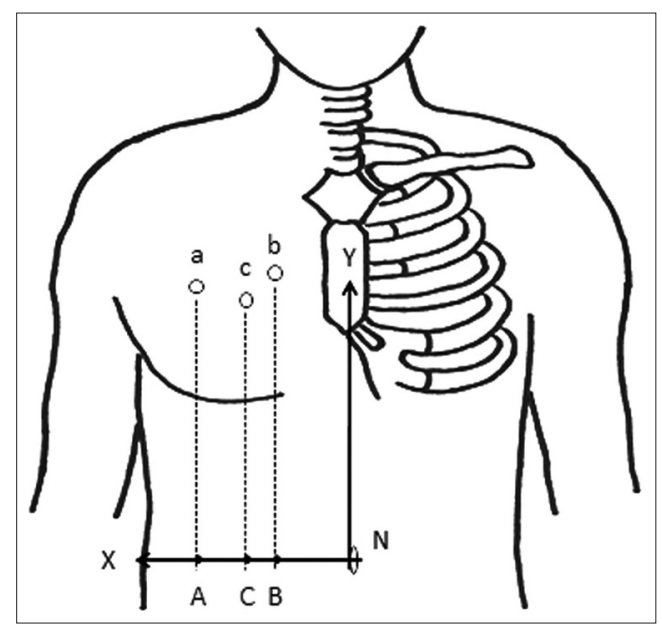

Figure 1: Diagram of acupuncture intervention. The $Y$-axis is parallel with the central line of trunk through the navel (N). The $X$-axis is the transverse line around the abdomen and lumbar region through the navel $(\mathrm{N})$. The needle insertion sites $\mathrm{A}, \mathrm{B}$, and $\mathrm{C}$ are corresponding to the pain spots $\mathrm{a}, \mathrm{b}$, and c separately acupuncture treatment. The result of NRS score declining up to 3 points was defined as "good pain relief." The duration of pain relief was also recorded. The secondary outcome was SMI lung volumes. ${ }^{[11,17]}$ These were evaluated through TriFlow, the incentive breathing spirometer, before and after the second and third acupuncture interventions. The numbers of patients with immediate increases and decreases in SMI lung volumes $10 \mathrm{~min}$ after the second and third acupuncture treatments were also recorded. The overnight sleep disturbance by pain of these patients was assessed with NRS (0-10), in which a score of 0 indicates perfect sleep quality and 10 indicates totally interrupted sleep by pain. Any complications or side effects during the treatment were recorded.

\section{Statistical analysis}

Statistical analyses were performed using SAS software version 9.2 (SAS Institute Inc., Cary, NC, USA). Demographic data between FN and control group including gender, age, and clinical characteristics were compared using the unpaired Student's $t$-test, one-way analysis of variance (ANOVA), and Chi-squared test. One-way ANOVA was performed for the changes in pain intensity, while two-way repeated measures ANOVA was performed for the comparisons of repeated measurements of pain relief percentages. The Spearman correlation coefficients were calculated for the correlations of factors that might be associated with pain intensity. Figures calculated were expressed as percentages and as mean \pm standard deviation. When the $p$ value was less than 0.05 , the result was considered statistically significant.

\section{RESULTS}

\section{Patient characteristics}

The trial flowchart is presented as Figure 2. From 8 December 2011 to 8 November 2012, 135 inpatients with rib fractures were assessed for eligibility. After screening, 92 inpatients met the inclusion criteria, with 58 of them participating in this study and randomly assigned to two groups. The demographic and clinical characteristics of the 58 recruited participants were analyzed. Fifty-two participants, including 27 patients in the FN group and 25 patients in the control group, completed the study and their efficacies were analyzed. One patient in the FN group and two controls discharged from the hospital early and could not receive complete treatment and assessment. Another patient in the FN group and one patient in the control group terminated the treatment due to fever of unknown origin. One patient in the control group left the study due to fear of acupuncture. The baseline characteristics of these participants are listed in Table 1, and there were no statistically significant differences between the two groups except for patient num- 


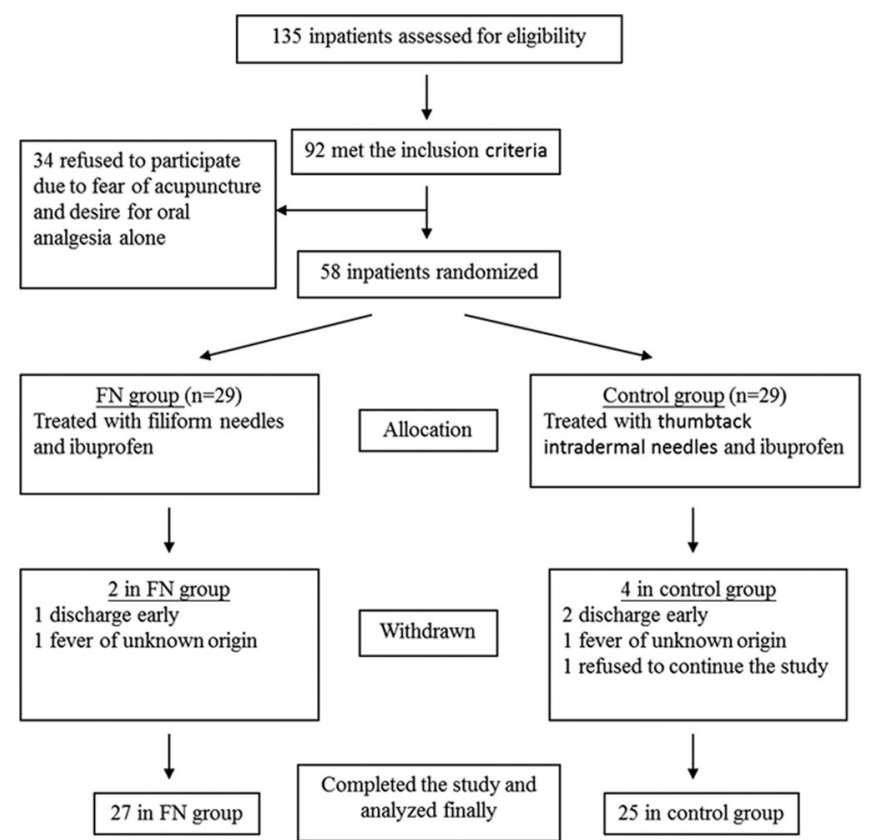

Figure 2: Trial flowchart

bers with chest tube insertion $(p=0.05)$. The initial pain intensity before the treatment in the two groups showed no significant difference $(p>0.05)$. All the patients received no extra analgesics, but ibuprofen.

The correlations of factors that might associate with pain intensity evaluation were also analyzed. It is shown that the number of fractured ribs was correlated with maximal pain after trauma and the initial pain intensity during deep breathing before treatment $(p<0.05)$, and had no correlation with initial pain intensity during coughing and turning over. ISS had correlation with initial pain intensity during deep breathing before treatment $(p<0.05)$ (data not shown).

\section{Primary outcome}

Table 2 represents the pain intensity and the degree of pain relief in patients during deep breathing, coughing, and turning over before and after each time of acupuncture treatment. The initial pain intensity under all assessed conditions before all three times of treatments showed no significant difference $(p>0.05)$ between the FN group and the control group, except for pain intensity during coughing before the third regimen $(p=0.05)$. After each time of treatment, there was statistically significant difference in pain intensity between the FN group and the control group under all assessed conditions $(p<0.05)$ except during deep breathing after the first and second treatments $(p>0.05)$. As for the improvement of pain after acupuncture intervention, the pain intensity in the FN group decreased more remarkably under every condition than the control group $(p<0.0001$ for deep breathing after first acupuncture intervention and for coughing and turning
Table 1: The demographic and clinical characteristics of the participants

\begin{tabular}{|c|c|c|c|}
\hline Characteristic & $\begin{array}{l}\text { FN group } \\
(n=29)\end{array}$ & $\begin{array}{l}\text { Control group } \\
\quad(n=29)\end{array}$ & $P$ value \\
\hline Age (years) & $52.7 \pm 15.2$ & $46.7 \pm 41.6$ & 0.12 \\
\hline Gender, M/F & $\begin{array}{l}19(65.5 \%) / \\
10(34.5 \%)\end{array}$ & $\begin{array}{c}21(72.4 \%) / \\
(27.6 \%)\end{array}$ & 0.57 \\
\hline BMI $\left(\mathrm{kg} / \mathrm{m}^{2}\right)$ & $25.4 \pm 4.7$ & $24.9 \pm 3.4$ & 0.65 \\
\hline Etiologies of trauma & & & 0.28 \\
\hline Traffic accident & $19(65.5 \%)$ & $20(69.0 \%)$ & \\
\hline Fall & $10(34.5 \%)$ & $7(24.1 \%)$ & \\
\hline Crush & $0(0 \%)$ & $2(6.9 \%)$ & \\
\hline Lesion side, R/L/B & $\begin{array}{c}12(41.4 \%) / \\
15(51.7 \%) / \\
2(6.9 \%)\end{array}$ & $\begin{array}{c}17(58.6 \%) / \\
11(37.9 \%) / \\
1(3.5 \%)\end{array}$ & 0.40 \\
\hline Number of fracture ribs & $3.6 \pm 1.1$ & $3.4 \pm 1.2$ & 0.41 \\
\hline ISS & $2.8 \pm 0.7$ & $2.8 \pm 0.7$ & 0.86 \\
\hline Complication & & & 0.20 \\
\hline Pneumothorax & $1(4.8 \%)$ & $5(26.3 \%)$ & \\
\hline Hemothorax & $6(28.6 \%)$ & $5(26.3 \%)$ & \\
\hline Hemopneumothorax & $5(23.8 \%)$ & $5(26.3 \%)$ & \\
\hline Chest tube insertion & $8(44.4 \%)$ & $13(76.5 \%)$ & 0.05 \\
\hline ICU days & $0.4 \pm 1.0$ & $0.2 \pm 0.8$ & 0.46 \\
\hline $\begin{array}{l}\text { Trauma to admission } \\
\text { (days) }\end{array}$ & $1.3 \pm 1.4$ & $0.9 \pm 1.1$ & 0.30 \\
\hline $\begin{array}{l}\text { Admission to } \\
\text { acupuncture (days) }\end{array}$ & $1.6 \pm 0.9$ & $1.6 \pm 0.9$ & 1.00 \\
\hline $\begin{array}{l}\text { Trauma to } \\
\text { acupuncture (days) }\end{array}$ & $2.9 \pm 1.7$ & $2.6 \pm 1.5$ & 0.42 \\
\hline $\begin{array}{l}\text { NRS of maximal pain } \\
\text { after trauma } \\
\text { NRS of initial pain } \\
\text { intensity before treatment }\end{array}$ & $9.0 \pm 1.3$ & $8.8 \pm 1.6$ & 0.58 \\
\hline Deep breath & $4.6 \pm 3.3$ & $4.2 \pm 2.8$ & 0.58 \\
\hline Cough & $7.8 \pm 2.3$ & $7.4 \pm 2.2$ & 0.60 \\
\hline Turnover & $7.9 \pm 2.2$ & $7.4 \pm 2.5$ & 0.45 \\
\hline Withdrawn & 2 & 4 & \\
\hline
\end{tabular}

All data are shown as $n(\%)$ or mean \pm SD. Abbreviations: F: Female; M: Male; BMI: Body mass index; R: Right; L: Left; B: Bilateral; ISS: Injury severity score; ICU: Intensive care unit; NRS: Numerical rating scale for pain measurement

over after all three interventions). The initial pain before the first intervention and the final pain after completing the third intervention had significant difference in both the groups $(p<0.0001$ in the FN group and $p<0.05$ in the control group) except for that assessed during turning over in the control group $(p=0.09)$. Furthermore, the initial pain intensities before all three interventions were significantly different in the FN group during coughing $(p=0.0004)$ and turning over $(p=0.0006)$ and in the control group during coughing $(p=0.0093)$

Table 3 shows that the percentages of pain relief under all assessed conditions before and after all three interventions had significant difference between the two groups $(p<0.0001)$. There are no significant differences within group $(p>0.05)$ or with interaction $(p>0.05)$ in 
Table 2: Pain intensity before and after treatment and change with NRS

\begin{tabular}{llcccc}
\hline $\begin{array}{l}\text { Action of } \\
\text { evaluation }\end{array}$ & Treatment & \multicolumn{4}{c}{ FN/control } \\
\cline { 3 - 6 } & & 1 & 2 & 3 & $p$ value, one-way ANOVA \\
\hline Deep & Pre & $4.6 \pm 3.3 / 4.2 \pm 2.8$ & $3.7 \pm 2.8 / 3.6 \pm 3.0$ & $2.9 \pm 2.3 / 3.2 \pm 2.5$ & $0.084 / 0.163$ \\
breath & Post & $2.3 \pm 2.3 / 3.5 \pm 2.7$ & $1.9 \pm 1.9 / 3.0 \pm 2.8$ & $1.3 \pm 1.4 / 2.6 \pm 2.5^{*}$ & $0.442 / 0.510$ \\
& Post-pre & $-2.3 \pm 1.8 / 0.7 \pm 0.9^{\ddagger}$ & $-1.9 \pm 1.6 / 0.6 \pm 0.9^{\dagger}$ & $-1.6 \pm 1.3 / 0.6 \pm 0.7^{*}$ & $0.0004 / 0.0093$ \\
Cough & Pre & $7.8 \pm 2.3 / 7.4 \pm 2.2$ & $6.5 \pm 2.2 / 7.2 \pm 2.1$ & $5.2 \pm 2.3 / 6.5 \pm 2.4^{*}$ & $0.288 / 0.255$ \\
& Post & $4.7 \pm 2.7 / 7.2 \pm 2.3^{*}$ & $3.9 \pm 2.4 / 7.0 \pm 2.3^{\ddagger}$ & $2.7 \pm 1.8 / 6.1 \pm 2.4^{\ddagger}$ & $0.0006 / 0.1233$ \\
& Post-pre & $-3.0 \pm 1.9 / 0.3 \pm 0.5^{\ddagger}$ & $-2.6 \pm 1.4 / 0.3 \pm 0.5^{\ddagger}$ & $-2.4 \pm 1.2 / 0.4 \pm 0.6^{\ddagger}$ & $0.399 / 0.436$ \\
& Pre & $7.9 \pm 2.2 / 7.4 \pm 2.5$ & $6.9 \pm 2.5 / 7.1 \pm 2.8$ & $5.3 \pm 2.6 / 6.4 \pm 3.0$ & $3.6 \pm 2.6 / 6.1 \pm 3.2^{*}$ \\
& Post & $5.2 \pm 3.1 / 7.2 \pm 2.9^{*}$ & $4.7 \pm 3.1 / 6.8 \pm 2.9^{*}$ & $-1.7 \pm 1.4 / 0.3 \pm 0.6^{\ddagger}$ & \\
\hline
\end{tabular}

All data are analyzed with 27 patients in the FN group and 25 in the control group, All data are shown as mean $\pm \mathrm{SD},{ }^{*} p<0.05,{ }^{\dagger} p<0.001,{ }^{\star} p<0.0001$ versus FN group, Abbreviations: Pre: The pain intensity evaluated before the treatment; Post: The pain intensity evaluated after the treatment; Postpre: The change of pain intensity after the treatment; NRS: Numerical rating scale

Table 3: Percentage of pain relief

\begin{tabular}{|c|c|c|c|c|}
\hline $\begin{array}{l}\text { Action of } \\
\text { evaluation }\end{array}$ & Treatment & FN group & Control group & $\begin{array}{c}p \text { value with two-way } \\
\text { repeated measures ANOVA }\end{array}$ \\
\hline \multirow[t]{4}{*}{ Deep breath (n1) } & 1 & $-48.72 \pm 19.69$ & $-16.58 \pm 18.47^{\stackrel{t}{*}}$ & \multirow{4}{*}{$<0.0001^{\S} 0.281^{\|} 0.711^{\pi}$} \\
\hline & 2 & $-48.29 \pm 24.66$ & $-20.72 \pm 27.03 *$ & \\
\hline & 3 & $-56.76 \pm 22.13$ & $-22.08 \pm 31.91^{\dagger}$ & \\
\hline & Pre 3 -pre $1 /$ pre 1 & -39.76 & -27.10 & \\
\hline \multirow[t]{4}{*}{ Cough (n2) } & 1 & $-40.86 \pm 25.05$ & $-4.21 \pm 8.25^{\ddagger}$ & \multirow{4}{*}{$<0.0001^{\S} 0.051^{\|} 0.291^{\pi}$} \\
\hline & 2 & $-43.52 \pm 22.57$ & $-5.20 \pm 9.42^{\ddagger}$ & \\
\hline & 3 & $-51.29 \pm 26.60$ & $-6.63 \pm 12.59^{\ddagger}$ & \\
\hline & Pre 3 -pre $1 /$ pre 1 & -34.42 & $-11.25^{*}$ & \\
\hline \multirow[t]{4}{*}{ Turnover (n3) } & 1 & $-34.69 \pm 27.80$ & $-7.53 \pm 14.74^{\ddagger}$ & \multirow{4}{*}{$<0.0001^{\S} 0.642^{\| 1} 0.950^{\llbracket /}$} \\
\hline & 2 & $-33.95 \pm 26.44$ & $-6.58 \pm 13.26^{\ddagger}$ & \\
\hline & 3 & $-37.19 \pm 30.64$ & $-8.42 \pm 15.89^{\dagger}$ & \\
\hline & Pre 3 -pre 1/pre 1 & -33.33 & $-12.26^{*}$ & \\
\hline
\end{tabular}

All data are shown as mean \pm SD (\%). Pre 3-pre 1/pre 1 represents the percentage of pain intensity change after two acupuncture interventions. n1, data are analyzed with 18 patients in the FN group and 20 in the control group; n2, data are analyzed with 26 patients in the FN group and 25 in the control group; $\mathrm{n} 3$, data are analyzed with 25 patients in the FN group and 25 in the control group. $* p<0.05,{ }^{*} p<0.001,{ }^{*} p<0.0001$ versus FN group; ${ }^{\S} p$ value between groups, " $p$ value within group, $" P$ value of interaction

both groups. After two times of treatments, the percentages of pain intensity change between the one before the third treatment and the original pain before the first treatment had significant difference between the two groups during coughing and turning over $(p<0.05)$, but not during deep breathing $(p>0.05)$. The numbers of patients analyzed are different because those patients with an initial pain intensity score of 0 before the first treatment were excluded from the analysis due to infeasibility of calculation.

The "good pain relief" assessment via acupuncture is illustrated in Table 4. Only one patient in the control group achieved "good pain relief" during deep breathing after the first and second regimens, while 8-17 patients in the FN group achieved "good pain relief" under any of the three assessed conditions after every treatment. There was statistically significant difference between the two groups during each assessed condition $(p<0.05$ after the second and third regimens in deep breathing and after the third regimen in
Table 4: Good pain relief via acupuncture interventions

\begin{tabular}{lccc}
\hline Treatment & \multicolumn{3}{c}{ Good pain relief (FN group/control group) } \\
\cline { 2 - 4 } & 1 & 2 & 3 \\
\hline Deep breath & $14(51.9) / 1(4.0)^{\ddagger}$ & $8(29.6) / 1(4.0)^{*}$ & $8(29.6) / 0(0)^{*}$ \\
Cough & $17(63.0) / 0(0)^{\ddagger}$ & $16(59.3) / 0(0)^{\ddagger}$ & $14(51.9) / 0(0)^{\ddagger}$ \\
Turnover & $14(51.9) / 0(0)^{\ddagger}$ & $11(40.7) / 0(0)^{\dagger}$ & $9(33.3) / 0(0)^{*}$ \\
\hline
\end{tabular}

All data are analyzed with 27 patients in the FN group and 25 in the control group. All data are shown as $n(\%) . * p<0.05,{ }^{\dagger} p<0.001,{ }^{\star} p<0.0001$

turning over, $p<0.001$ after the second regimen in turning over, other assessments had $p<0.0001$ ).

As for the duration of pain relief, 22 of 27 inpatients (81.5\%) in the FN group and 10 of 22 controls (40.0\%) revealed that the pain relief effect of acupuncture maintained for at least $6 \mathrm{~h}$, while other participants were not aware of it. Fifteen of 27 patients $(55.6 \%)$ in the FN group indicated that pain intensity increased gradually after removing the needles. 


\section{SMI lung volumes}

Table 5 shows the SMI lung volumes in secondary outcome variables. After the second acupuncturing, the results revealed a statistically significant improvement in the immediate SMI lung volumes. The FN group had an average of immediate SMI increase of $142.60 \pm 204.10 \mathrm{ml}$, while the control group had an increase of $6.00 \pm 143.10 \mathrm{ml}(p<0.01)$. However, no significant improvement was observed after the third treatment (FN group SMI $50.00 \pm 124.0 \mathrm{ml}$, control group SMI $10.00 \pm 35.36 \mathrm{ml} ; p>0.05$ ).

It is noteworthy that after the second acupuncturing, 15 of 27 patients in the FN group had immediate SMI increase (average $276.7 \mathrm{ml}$ ) in contrast to only 3 controls with immediate SMI increase (average $216.7 \mathrm{ml})(p=0.001)$. Also, one patient from the FN group and two patients from the control group had immediate decrease of SMI (FN group: $300 \mathrm{ml}$; control group: $400 \mathrm{ml}$ in average) after the second acupuncturing (no statistical significance), while others remained the same. However, no significant difference was observed between the two groups in patient numbers with SMI increase after the third treatment $(p>0.05)$, with two patients in the FN group having immediate decrease of SMI (average $-125 \mathrm{ml}$ ).

\section{Sleep quality}

There was no statistically significant difference in the baseline overnight sleep disturbance between the two groups as shown in Table 6. Among the acupuncture interventions, significant improvement was shown only after the first intervention $(p<0.05)$ but not after the second intervention $(p=0.53)$. The overnight sleep disturbance after the third intervention could not be assessed due to the limited consented observation period in this study. The sleep quality improvement after two acupuncture interventions had no significant difference between the two groups $(p>0.05)$.

\section{Side effects}

Two patients in the FN group complained about intermittent, uncomfortable, but endurable sting sensation at the insertion sites of filiform needles. One patient from the control group complained of itch due to original sensitive skin and adhesive tape, and the condition was resolved after changing the material of the tape. Five patients in the FN group had mild hemorrhage when removing the needles, which had left some ecchymoses at the needle sites with no swelling or pain. There were no patients dropping out because of side effects. No patients removed the needles before the planned needle retention time of $6 \mathrm{~h}$.

\section{DISCUSSION}

This study is a pilot trial that first addresses the efficacy of acupuncture as a complementary therapy to ease
Table 5: SMI lung volumes (ml)

\begin{tabular}{|c|c|c|c|c|c|}
\hline & & eatment & FN group & Control group & $p$ value \\
\hline SMI lung & 2 & Pre & $753.7 \pm 280.8$ & $774.0 \pm 227.8$ & 0.78 \\
\hline volumes & & Post & $896.3 \pm 213.0$ & $768.0 \pm 228.2$ & 0.04 \\
\hline & & Post-pre & $142.60 \pm 204.10$ & $-6.00 \pm 143.10$ & $<0.01$ \\
\hline & 3 & Pre & $883.3 \pm 229.1$ & $902.0 \pm 185.7$ & 0.75 \\
\hline & & Post & $933.3 \pm 214.4$ & $912.0 \pm 193.3$ & 0.71 \\
\hline & & Post-pre & $50.00 \pm 124.0$ & $10.00 \pm 35.36$ & 0.12 \\
\hline Immediate & 2 & Increase & 15 (276.7) & $3(216.7)$ & 0.001 \\
\hline SMI change & & Decrease & $1(-300)$ & $2(-400)$ & 0.51 \\
\hline & 3 & Increase & 7 (228.6) & $2(125.0)$ & 0.09 \\
\hline & & Decrease & $2(-125)$ & $0(0)$ & 0.17 \\
\hline
\end{tabular}

All data are shown as mean \pm SD or $n$ (volumes in average). Abbreviations: SMI: Sustained maximal inspiration; Pre: The SMI evaluated before the treatment; Post: The SMI evaluated after the treatment; Post-pre: The change of SMI lung volumes after the treatment

Table 6: Overnight sleep disturbance by pain

\begin{tabular}{lccc}
\hline $\begin{array}{l}\text { Assessment } \\
\text { time }\end{array}$ & $\begin{array}{c}\text { FN group } \\
(n=27)\end{array}$ & $\begin{array}{c}\text { Control group } \\
(n=25)\end{array}$ & $p$ value \\
\hline Pre-intervention & $4.37 \pm 4.33$ & $4.84 \pm 3.90$ & 0.68 \\
Post 1 & $2.56 \pm 2.95$ & $4.48 \pm 3.77$ & 0.04 \\
Post 2 & $2.04 \pm 2.56$ & $2.52 \pm 2.89$ & 0.53 \\
Post 2-pre & $-2.33 \pm 4.28$ & $-2.32 \pm 3.11$ & 0.99 \\
\hline
\end{tabular}

All data are shown as mean \pm SD. Abbreviations: Pre-intervention: Assessment of sleep disturbance during the last night before the first treatment; Post 1: Assessment of overnight sleep disturbance after the first treatment; Post 2: Assessment of overnight sleep disturbance after the second treatment; Post 2-pre: The change between pre-intervention and post 2

rib fracture pain, which has never been explored before. The intolerable pain of inpatients with rib fractures usually reaches a peak and then is relieved in 2-4 weeks after trauma under the use of analgesia. ${ }^{[1,18]}$ While acute pain control during hospitalization has been well studied, there still exists room for improvement, and better therapeutic strategies other than conventional oral analgesics and nerve blocker injection are to be explored..$^{[1,5,10,17]}$

The inpatients who met the inclusion criteria and had maximal pain intensity of more than 5 with the NRS and responded poorly to pain control via pharmacological treatment were screened and enrolled. We aimed to evaluate the efficacy and efficiency of an innovative form of acupuncture intervention in reducing pain in these subjects.

This study adopted two different kinds of needles, filiform needles and TI needles, via the same acupuncture modality to treat rib pain. Filiform needles are commonly applied in acupuncture for pain relief perpendicularly in most practitioners' experience and acupuncture studies. ${ }^{[15,16]}$ According to our clinical experience, inserting the needles at an angle of 15 could offer better effect on rib pain relief. On the contrary, TI needles, which are generally regarded as ear needles, are usually applied in small and shallow 
auricular acupuncture points due to their shorter length of needles and could relieve various kinds of distal pain, except post-fracture pain. ${ }^{[19-22]}$ In this study, while the filiform needles penetrate into the skin with deeper stimulation depth and length, TI needles were only placed lightly upon the skin surface of the patients' fleshy abdomen or flank to limit their acting dose of treatment and, hence, their efficacy. Therefore, distinct from their usual function in auricular acupuncture, the TI needles were used as control or placebo in this study in contrast with filiform needles.

Considering overall results, the FN group showed more effective pain relief than the control group during activities of deep breathing, coughing, and turning over in bed. The pain intensity could even decline up to 3 points immediately after acupuncturing with filiform needles, which oral analgesia could not achieve. Although the control group showed a tendency of slight pain relief, the significant efficacy of acupuncture using FN needle for pain relief was still illustrated through subjective pain assessment by the patients. Besides, the effect of natural recovery from rib reunion in reducing pain in both groups should also be taken into consideration.

It is shown that the pain intensity of inpatients with rib fractures during activities of deep breathing, coughing, and turning over was reduced after FN acupuncture intervention. It is noteworthy that fearlessness in taking deep breath could decrease the rate of pulmonary complications and increase the desire for ventilation training. ${ }^{[3]}$ Moreover, some patients in the FN group mentioned about better sputum expectoration, and also, some patients indicated the elevated motivation to get up and move when acupuncture eased the pain. It implied that the therapy applied reduced the inconvenience due to trauma and improves the life quality of the patients during hospitalization.

Overall, the initial pain induced by deep breathing scored less than that induced by coughing or turning over. Thus, significant improvement of pain control in coughing and turning over was demonstrated by the percentage of pain relief rather than the difference in pain intensity before and after the treatment. As a matter of fact, a diversity of pain intensity assessed in the patients with rib fractures during different actions was observed. In general, the motion of deep breathing is taken at rest, while the action of coughing might implicate sudden and intense expiration of chest cavity, and turning over the body requires the rotation of trunk and extremities. However, our results showed little correlation between the pain intensity during coughing or turning over and the number of fractured ribs. It implies that the pain sensation of patients is subjective, and the cause of pain might not only be the fractured ribs but also the injured soft tissue around the trauma or other undefined factors. After three times of acupuncture interventions with this modality, significant pain relief was shown during all the ac- tions observed in the FN group, indicating that acupuncture benefits mainly the pain sensation of the patients, regardless of the number of fractured ribs or the severity of trauma.

The increase in SMI was only observed in patients with lower lung volumes through the treatment, while others with original SMI close to $1200 \mathrm{ml}$ showed no obvious improvement. Many patients had the same or even decreased SMI lung volumes after acupuncturing. In contrast with the studies of Haenel et al. ${ }^{[11]}$ and Truitt et al..${ }^{[17]}$ which reported decreased pain with increased SMI under continuous intercostal nerve blocker, it was concluded that pain relief via acupuncture with this novel modality acts through different mechanisms than analgesia. In comparisons of the implement of continuous nerve blocker provider, acupuncture intervention was less invasive and did not interfere the function of respiratory muscles.

The sleep quality did not improve through every acupuncture intervention. However, this does not necessarily mean that the treatments did not have an effect on the patients' quality of sleep. All the participants received acupuncture in the morning, so the needles were removed before 6 o'clock in the evening. However, the effect might be influenced by different treatment times. Moreover, this study cannot distinguish the cause of sleep disturbances, whether being caused by pain or other preexisting factors.

A fine feature in this trial was the active evaluation, which is advocated in some studies. ${ }^{[13,23]}$ In contrast with the static assessment in earlier pain-related studies, evaluation of pain intensity during some pain-inducing actions was performed. Clinical experience in treating patients with multiple rib fractures shows that less pain is felt when patients stay inactive. Therefore, pain intensity evaluation is more accurate when patients are performing specific motions which healthy people could perform with ease in normal life. When the goal of treatment is to decrease the disability of these inpatients, it is pain that disturbs their recovery for performing normal function, which should first be relieved.

An innovative acupuncture modality was performed in this study. The acupoints located in non-diseased areas were chosen corresponding to the pain spots, which are different from "Ah shi point." In addition, compared to the traditional acupuncture studies that administered immobile sets of acupuncture points, ${ }^{[15,24,25]}$ this model could adapt to the great diversity of pain sites in patients with rib fractures with repeatable efficacy.

While administering the acupuncture modality, a remarkable phenomenon of shifted pain spots was observed in the FN group. After inserting the first needle at the first point, the initial pain spot disappeared immediately, which was followed by manifestation of the second pain spot for orientating the second acupoint. The sites of the five pain spots and, hence, the five acupoints were diverse in every 
patient, located from the front side to the backside of the patients' trunks, depending on the location of the trauma. Only five needles were inserted in each patient's body, and $30-50 \%$ of pain relief could be reached in the study. Though the patients still felt pain after the treatment, the pain intensity might decrease through further acupuncture with this model. In the control group, some patients showed similar diversity in pain spot locations, while the pain spots of others remained the same after the insertion of one needle, leading to the following needles inserted at the same point.

Two kinds of devices were taped and fixed on the points for $6 \mathrm{~h}$ to investigate the duration of pain relief. In modern acupuncture treatment, the retention of the needles usually lasted for 15-20 min, but according to our clinical experience, short-time retention provides limited effect for patients with rib fractures who have severer pain than those who have the other general pain diseases. The 6-h fixation was achievable when the filiform needles were inserted almost horizontally into the skin, while TI needles were originally designed to treat for long-time retention. Besides, because of the pain relief effect, most patients showed high compliance to this modality. Furthermore, the risk of bending or breaking the needle was low because patients with rib fractures usually have limited body movement due to pain. Therefore, the technique of long-time retention of filiform needles for pain relief was safe and feasible.

Although this acupuncture modality produced immediate pain relief that lasted for at least $6 \mathrm{~h}$ in the FN group, some patients experienced a rebound of pain that even reached the original pain intensity immediately or within a couple of hours after removing the needles. Some controls were not aware of the duration of pain relief, which may be possible due to the unfelt efficacy of TI needles. We can, therefore, assume that the effect of pain relief of filiform needles could be prolonged and further consolidated if a better model of long-time retention of filiform needles is developed in further studies.

Still, there were some difficulties when performing the acupuncture modality. For example, if the pain spot was located in the backside of the trunk making it difficult for the patient to point the accurate site of the pain spot, it would lead to the deviation of acupoint and disrupt the therapeutic effect. Also, for the patients who are hurt in a large area of the flank, only five acupoints would offer limited outcomes. Moreover, patients who had diffused pain and could not find definite pain spot would not adapt to this acupuncture model.

The timing, duration, and retention of the acupuncture treatment might impact analgesia efficacy. The period from trauma to treatment of every patient could not be consistently controlled clinically. According to the Kerr-Valentic et al.' ${ }^{[1]}$ study, patients with rib fracture have an average of five hospitalization days. However, the actual length of hospital stay varies with several factors such as willingness of the inpatients and care problems, which are unrelated to pain intensity, so we could not define an endpoint to evaluate the overall efficacy of pain relief by acupuncture through the hospital stay. Furthermore, continued research is required to better understand if models of long-time retention such as this acupuncture modality and Fu's subcutaneous needling could provide longer analgesia effect. ${ }^{[26]}$

The mechanism of acupuncture analgesia has been studied extensively in some traditional acupuncture researches. ${ }^{[27-30]}$ Physiological and neural mechanisms such as serotonergic systems and some neurotransmitters are involved when considering official acupoints and meridians. In contrast, our study adopted an innovative acupuncture modality, focusing on various acupuncture points orientated by patients' pain spots, which did not locate along regular meridians as in the traditional acupuncture treatment. Therefore, the current hypotheses to explain how our treatment could relieve rib pain are not applicable. The efficacy of this acupuncture modality has been presented in this study, and its potential mechanisms, whether by enhancing the effect of anti-inflammatory medications or by exerting its own therapeutic effect, require further analysis, which may challenge the current theory and provide a new version of acupuncture treatment.

\section{Conclusions}

This novel modality of acupuncture adjunctive to ibuprofen appears to be a safe and viable therapy for the inpatients with multiple rib fractures. The use of filiform needles produced more obvious benefit of acute pain relief, which persisted for at least $6 \mathrm{~h}$, than the use of TI needles when the evaluation was made during deep breathing, coughing, and turning over the body. Further studies will be designed to investigate the efficacy of this modality compared with routine therapy and real sham acupuncture in relieving pain due to rib fractures.

\section{Acknowledgments}

The authors would like to thank all the staff of the Department of Trauma and Emergency Surgery for providing and caring the study patients and collecting data. The authors also wish to acknowledge the help of Miss. Han-Fang Cheng, Mr. Pi-Chiang Hsu, and Mr. Tyson Scott Skriver in giving advice of revision and Dr. Hsing-Yu Chen in the suggestion of research.

\section{REFERENCES}

1. Kerr-Valentic MA, Arthur M, Mullins RJ, Pearson TE, Mayberry JC. Rib fracture pain and disability: Can we do better? J Trauma 2003;54:1058-64. 
2. Simon BJ, Cushman J, Barraco R, Lane V, Luchette FA, Miglietta M, et al. Pain management guidelines for blunt thoracic trauma. J Trauma 2005;59:1256-67.

3. Karadayi S, Nadir A, Sahin E, Celik B, Arslan S, Kaptanoglu M. An analysis of 214 cases of rib fractures. Clinics 2011;66:449-51.

4. D'Arcy Y. Easing pain from blunt thoracic trauma. Nursing 2005;35:17.

5. Karmakar MK, Ho AM. Acute pain management of patients with multiple fractured ribs. J Trauma 2003;54:615-25.

6. Liman ST, Kuzucu A, Tastepe AI, Ulasan GN, Topcu S. Chest injury due to blunt trauma. Eur J Cardiothorac Surg 2003;23:374-8.

7. Sirmali M, Turut H, Topcu S, Gulhan E, Yazici U, Kaya S, et al. A comprehensive analysis of traumatic rib fractures: Morbidity, mortality and management. Eur J Cardiothorac Surg 2003;24:133-8.

8. Winters BA. Older adults with traumatic rib fractures: An evidence-based approach to their care. J Trauma Nurs 2009;16:93-7.

9. Mayberry JC, Kroeker AD, Ham LB, Mullins RJ, Trunkey DD. Long-term morbidity, pain, and disability after repair of severe chest wall injuries. Am Surg 2009;75:389-94.

10. Ho AM, Karmakar MK, Critchley LA. Acute pain management of patients with multiple fractured ribs: A focus on regional techniques. Curr Opin Crit Care 2011;17:323-7.

11. Haenel JB, Moore FA, Moore EE, Sauaia A, Read RA, Burch JM. Extrapleural Bupivacaine for Amelioration of Multiple Rib Fracture Pain. J Trauma 1995;38:22-7.

12. Nirula R, Diaz JJ Jr, Trunkey DD, Mayberry JC. Rib fracture repair: Indications, technical issues, and future directions. World J Surg 2009;33:14-22.

13. Kotani N, Hashimoto H, Sato Y, Sessler DI, Yoshioka H, Kitayama M, et al. Preoperative intradermal acupuncture reduces postoperative pain, nausea and vomiting, analgesic requirement, and sympathoadrenal responses. Anesthesiology 2001;95:349-56.

14. Paley CA, Johnson MI. Acupuncture for cancer-induced bone pain: A pilot study. Acupunct Med 2011;29:71-3.

15. Mavrommatis CI, Argyra E, Vadalouka A, Vasilakos DG. Acupuncture as an adjunctive therapy to pharmacological treatment in patients with chronic pain due to osteoarthritis of the knee: A 3-armed, randomized, placebo-controlled trial. Pain 2012;153:1720-6.

16. Vas J, Aranda JM, Modesto M, Benitez-Parejo N, Herrera A, Martinez-Barquin DM, et al. Acupuncture in patients with acute low back pain: A multicentre randomized controlled clinical trial. Pain 2012;153:1883-9.
17. Truitt MS, Mooty RC, Amos J, Lorenzo M, Mangram A, Dunn E. Out with the old, in with the new: A novel approach to treating pain associated with rib fractures. World J Surg 2010;34:2359-62.

18. Karangelis D, Tagarakis G, Karkos C, Pantelaki I, Desimonas N, Papadopoulos D, et al. Rib fractures and pain peak 2 weeks down the line: Myth or a fact? Am J Emerg Med 2011;29:229-38.

19. Sator-Katzenschlager SM, Scharbert G, Kozek-Langenecker SA, Szeles JC, Finster G, Schiesser AW, et al. The short- and long-term benefit in chronic low back pain through adjuvant electrical versus manual auricular acupuncture. Anesth Analg 2004;98:1359-64.

20. Usichenko TI, Dinse M, Hermsen M, Witstruck T, Pavlovic D, Lehmann Ch. Auricular acupuncture for pain relief after total hip arthroplasty - a randomized controlled study. Pain 2005;114:320-7.

21. Wang SM, Dezinno P, Lin EC, Lin H, Yue JJ, Berman MR, et al. Auricular acupuncture as a treatment for pregnant women who have low back and posterior pelvic pain: A pilot study. Am J Obstet Gynecol 2009;201:271.e1-9.

22. Hunter RF, McDonough SM, Bradbury I, Liddle SD, Walsh DM, Dhamija $\mathrm{S}$, et al. Exercise and auricular acupuncture for chronic low-back pain: A feasibility randomized-controlled trial. Clin J Pain 2012;28:259-67.

23. Kehlet H, Dahl JB. Assessment of postoperative pain--need for action!. Pain 2011;152:1699-700.

24. Vas J, Perea-Milla E, Mendez C, Sanchez Navarro C, Leon Rubio JM, Brioso M, et al. Efficacy and safety of acupuncture for chronic uncomplicated neck pain: A randomised controlled study. Pain 2006;126:245-55.

25. Liang Z, Zhu X, Yang X, Fu W, Lu A. Assessment of a traditional acupuncture therapy for chronic neck pain: A pilot randomised controlled study. Complement Ther Med 2011;19(Suppl 1):S26-32.

26. Zhonghua F, Ryan S. Fu's Subcutaneous needling, a modern style of ancient acupuncture? Acupuncture in modern medicine. In: Lucy LC, Tsung OC, editors. UK: InTech; 2013. p. 179-203.

27. Irnich D, Beyer A. Neurobiological mechanisms of acupuncture analgesia. Schmerz 2002;16:93-102.

28. Han JS. Acupuncture and endorphins: Mini review. Neurosci Lett 2004;361:258-61.

29. Audette JF, Ryan AH. The role of acupuncture in pain management. Phys Med Rehabil Clin 2004;15:749-72.

30. David B. Mechanisms of acupuncture. Medical Acupuncture: A Western Scientific Approach. In: Filshie J, White A, editors. London: Churchill Livingstone; 1998. p. 69-82. 